\title{
Thermal and hydraulic calculation in a model of industrial branched pipeline transporting a single-phase liquid flow
}

\author{
S. A. Mayakova ${ }^{1,}{ }^{*}$ and L. Y. Privalov ${ }^{1}$ \\ ${ }^{1}$ Ufa State Aviation Technical University, Ufa, Russia
}

\begin{abstract}
This paper proposes a system of differential equations that describes changes of pressure and temperature of a stationary liquid flow through straight, turning, and branched pipeline sections. We developed a program, using the proposed system of equations, that carries through hydraulic and thermohydraulic calculations, allows to choose diameter of various pipeline systems. The key feature of the program is the ability to solve monophasic problems such as: friction losses; local resistance losses; heat dissipation into the environment; flow distribution.
\end{abstract}

\section{Introduction}

Hydraulic and thermal calculation of branched systems of pipelines is a complicated engineering and technological problem that is linked to computation of performance characteristics (pressure losses, temperature losses; evaluating consumption, pressure, and temperature in all junctions and resistances) of an existing or a proposed pipeline system with known geometrical and structural parameters. The task of developing universal mathematical models and numerical algorithms of analysis of monophasic flows in complex systems of pipelines and their implementation as a computer program is relevant and holds a practical significance since it is in demand in various industries: petrochemical, atomic, oil and gas production etc. A large number of recent scientific works is dedicated to this task, for example, [1-6].

The goal of this work is a development of an effective program suitable for modeling of a stationary monophasic liquid flow in a branched pipeline system (figure 1).

\section{Model selection and discretization scheme}

\subsection{Model of stationary single phase liquid flow}

The program is based on the model of hydraulic calculation of stationary flow properties (1) that is a modification on the model proposed in [3,4]. The model is written down in measurable thermodynamic variables 'pressure' and 'temperature', and is completed with the

\footnotetext{
* Corresponding author: Mayakovasa@mail.ru
} 
state equation (2) (from [7]). Presented model (1)-(2) is now applied to differently oriented straight pipeline sections, turnings, and sections of narrowing and widening.

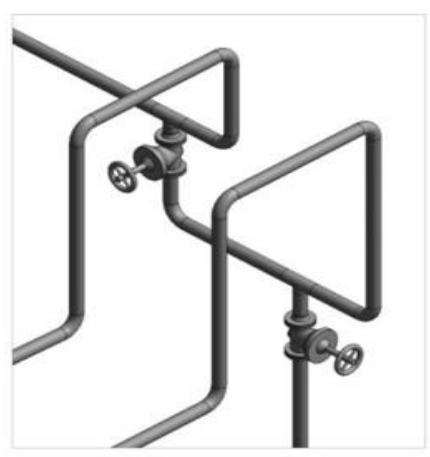

a

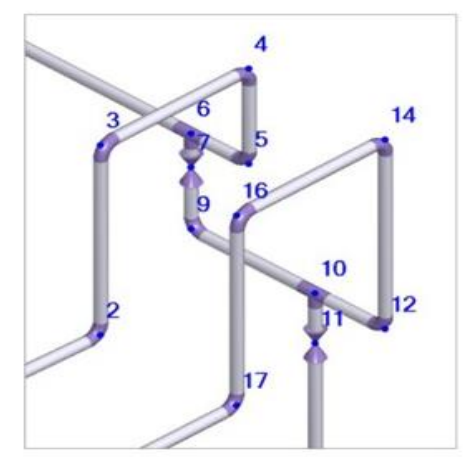

b

Fig. 1. An example of a branched part of an industrial pipeline: a - actual representation, $b$ structural model.

$$
\begin{aligned}
& \left\{\begin{array}{l}
\frac{d p}{d l}=\frac{1}{\left[1-M^{2}\right]}\left(\left[\left.\frac{u}{T} G^{2} \frac{d u}{d s}\right|_{p}+1\right]\left(\frac{d p}{d l}\right)_{f}+\left.G \frac{d u}{d s}\right|_{p} \frac{1-\frac{T_{e n v}}{T}}{A}-\frac{g \sin \theta}{u}\right) \\
\frac{d T}{d l}=\frac{1}{c_{p}}\left(\left(\frac{d u}{d T}\right)_{p} T \frac{d p}{d l}-u \frac{d p_{f}}{d l}-\frac{q}{A G}-u \frac{d p_{\text {local }}}{d l}\right)
\end{array}\right. \\
& M=G\left(-\left.\frac{d u}{d p}\right|_{s}\right)^{0.5},\left.\quad\left(\frac{d u}{d s}\right)\right|_{p}=\left.\left(\frac{d u}{d T}\right)\right|_{p} \frac{T}{c_{p}},\left.\quad\left(\frac{d u}{d p}\right)\right|_{s}=\left.\left(\frac{d u}{d p}\right)\right|_{T}+\left.\frac{T}{c_{p}}\left(\frac{d u}{d T}\right)\right|_{p} ^{2}, \\
& \left.\left(\frac{d u}{d p}\right)\right|_{T}=-\beta u_{0},\left.\quad\left(\frac{d u}{d T}\right)\right|_{p}=\alpha u_{0} \\
& u=\frac{1}{m}\left(a+b T-a_{1} p-c p T+c_{1} p^{2}+c_{2} p T^{2}+V_{e} e^{-a_{2} p-\frac{E+a_{3} p}{R T}}\right) .
\end{aligned}
$$

In the equations above $\mathrm{p}$ - pressure [Pa]; 1 - length from the beginning of the pipe [m]; $\mathrm{G}$ - mass flow $[\mathrm{kg} /(\mathrm{m} 2 \mathrm{~s})] ; \mathrm{u}$ - specific volume $[\mathrm{m} 3 / \mathrm{kg}] ; d p_{f} / d l$ - pressure loss per unit of length due to the friction $[\mathrm{Pa} / \mathrm{m}] ; \theta$ - pipe elevation angle relative to the horizon [rad]; $d p_{\text {local }} / d l$ - local resistance losses that appear in a narrowing or a widening, when branching) per unit of length $[\mathrm{Pa} / \mathrm{m}] ; q$ - heat loss of the substance into the environment per unit of length $[\mathrm{J} / \mathrm{m}] ; \mathrm{A}-$ area of the cross-section of the pipe $[\mathrm{m} 2] ; \mathrm{s}-$ specific entropy of the mixture $[\mathrm{J} /(\mathrm{kg} \mathrm{K})] ; \mathrm{T}$ - temperature $[\mathrm{K}] ; T_{\text {env }}$ - environment temperature $[\mathrm{K}] ; u_{0}-$ specific volume at $\mathrm{T}=0 \mathrm{~K}[\mathrm{~m} 3 / \mathrm{kg}] ; \alpha$ - isobaric coefficient of expansion; $\beta$ - isothermal coefficient of expansion; $\mathrm{M}$ - Mach number; $c_{p}$ - specific heat capacity $[\mathrm{J} /(\mathrm{kg} \mathrm{K})]$; $a, a_{1}, a_{2}, a_{3}, b, c, c_{1}, c_{2}, E, V_{e}$ - approximation constants (see [7]); R - gas constant; $\mathrm{m}$ mass $[\mathrm{kg}]$.

\subsection{Discretization scheme and length step selection}

The model (1)-(2), having been supplemented with boundary conditions, was discretized using Euler and Runge-Kutta schemes and implemented in the program. The numerical error for both methods was evaluated. The calculations have shown that, while having the length 
of the pipeline section $\mathrm{L}$ of around $1000 \mathrm{~m}$, starting with $\mathrm{n}=1000$, the relative difference between solutions obtained using $\Delta l_{n}$ and $\Delta l_{n+1}$, where $\Delta l_{n}=L / n$, was roughly 10-6 for the Euler method and 10-12 for the Runge-Kutta method, going lower with the increase of $n$. That said, the chosen length step $\Delta l=1 \mathrm{~m}$ allows for an acceptable numerical precision.

\subsection{Calculation of the pressure losses due to the friction and local resistances}

Discretized model (1)-(2) contains quantities $\Delta p_{f} / \Delta l$ and $\Delta p_{\text {local }} / \Delta l$, that define pressure losses and losses due to local resistances, such that appear in the places of narrowing, widening or branching. The derivation of such quantities is described below.

The pressure losses due to the friction in a laminar flow inside a round pipe can be described using Darcy-Weisbach equation [8-9]:

$$
\Delta p_{f}=\gamma \rho l \frac{G^{2}}{2 A^{\prime}}
$$

where 1 - the length of pipe section, $\gamma$ - the Darcy friction factor, $\rho$ - fluid pressure.

Pressure losses upon a sudden widening (figure 2a) are described using Borda-Carnot equation [9]:

$$
\Delta p_{12}=\rho \zeta_{12} \frac{G_{2}^{2}}{2}
$$

where $\zeta_{12}=(1-\varepsilon)^{2}-$ sudden widening coefficient, $\varepsilon=A_{1} / A_{2}-$ jet compression coefficient.

Similarly, upon sudden narrowing (figure $2 b$ ):

$$
\Delta p_{21}=\rho \zeta_{21} \frac{G_{1}^{2}}{2}
$$

where $\zeta_{21}=\left(\varepsilon^{-1}-1\right)^{2}-$ sudden narrowing coefficient.

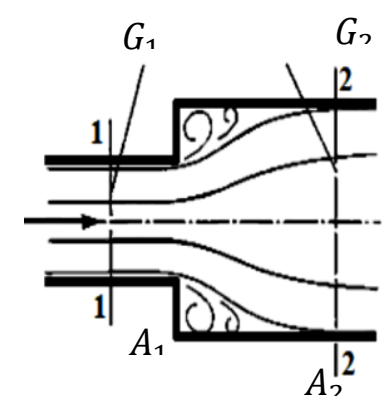

a

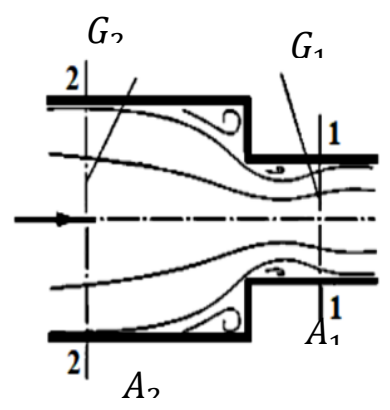

$\mathrm{b}$

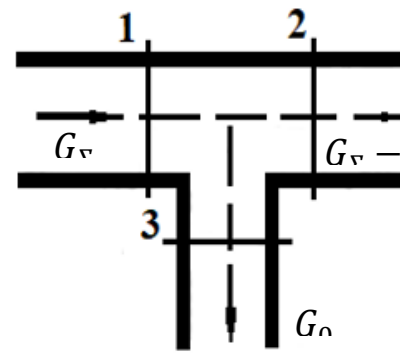

C

Fig. 2. Types of local resistances: a - sudden widening, b - sudden narrowing, c - branching.

In the places of branching (figure $2 \mathrm{c}$ ) pressure losses along primary passage between sections 1 and 2 are calculated using

$$
\Delta p_{12}=\xi_{12} \rho \frac{\left(G_{\Sigma-} G_{0}\right)^{2}}{2}
$$


and in the offshoot between sections 1 and 3 using

$$
\Delta p_{13}=\xi_{13} \rho \frac{G_{0}^{2}}{2}
$$
[8].

where $\xi_{12}, \xi_{13}-$ resistance coefficients between sections 1 and 2 , and 1 and 3 respectively

\section{Program testing}

Three models of various sections of industrial pipelines were designed in order to test the program (as in tests from [3]). Ambient temperature was $T_{e n v}=293 \mathrm{~K}$. The working fluid was water.

Constants for the fluid state equation (2) for water were taken from [7] and are provided in the table 1. Authors of the work [7] specify the valid range for the approximation (2) that lies within the interval of $0.1-100 \mathrm{MPa}$ for the pressure and $273-623 \mathrm{~K}$ for the temperature.

Table 1. Fluid state equation (2) constant values for water.

\begin{tabular}{|l|c|c|c|c|c|}
\hline Constant & $a$ & $a_{1}$ & $a_{2}$ & $a_{3}$ & $b$ \\
\hline Value & $9.4510^{-4}$ & $4.810^{-8}$ & $4.410^{-4}$ & $7.110^{-4}$ & $1.6110^{-7}$ \\
\hline Constant & $c$ & $c_{1}$ & $c_{2}$ & $E$ & $V_{e}$ \\
\hline Value & 0 & 0 & $1.610^{-13}$ & 18.5 & $1.510^{-2}$ \\
\hline
\end{tabular}

\subsection{Test 1: section with turns, narrowings and widenings}

The test model for the first example is a pipeline section that contains 16 straight pipes (vertical and horizontal), 9 turns and 6 parts with sudden narrowing or widening (figure 3 ). Initial pressure was set to $p_{0}=0.2 \mathrm{MPa}$, initial temperature was $T_{0}=333 \mathrm{~K}$, mass flow $\mathrm{G}$ $=117.37 \mathrm{~kg} /(\mathrm{m} 2 \mathrm{~s})$, diameter of straight pipes $\mathrm{d}=0.301 \mathrm{~m}$.

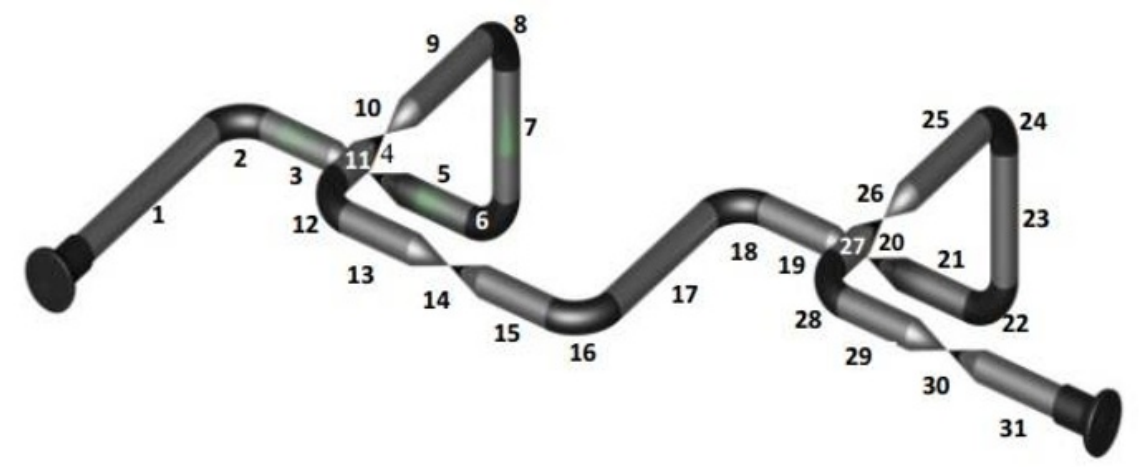

Fig. 3. The section of an industrial pipeline for the test 1.

For the first test, using Runge-Kutta method, the losses of pressure and temperature along each of the isolated parts of the pipeline were calculated (figure 4, 5). The figures show that the largest leaps of pressure losses correlate to the parts with sudden widenings or narrowings of the pipe diameter. And the largest temperature losses on the parts with widening/narrowing and turning. 


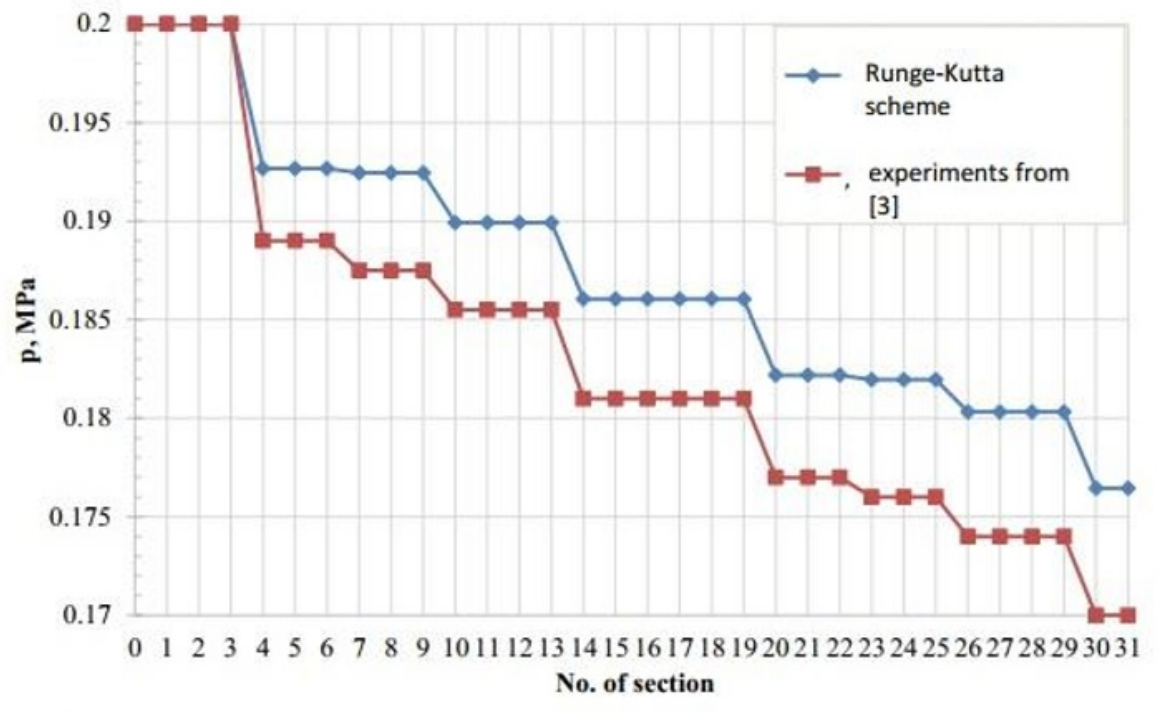

Fig. 4. Fluid pressure losses inside isolated parts of the pipeline for the test 1.

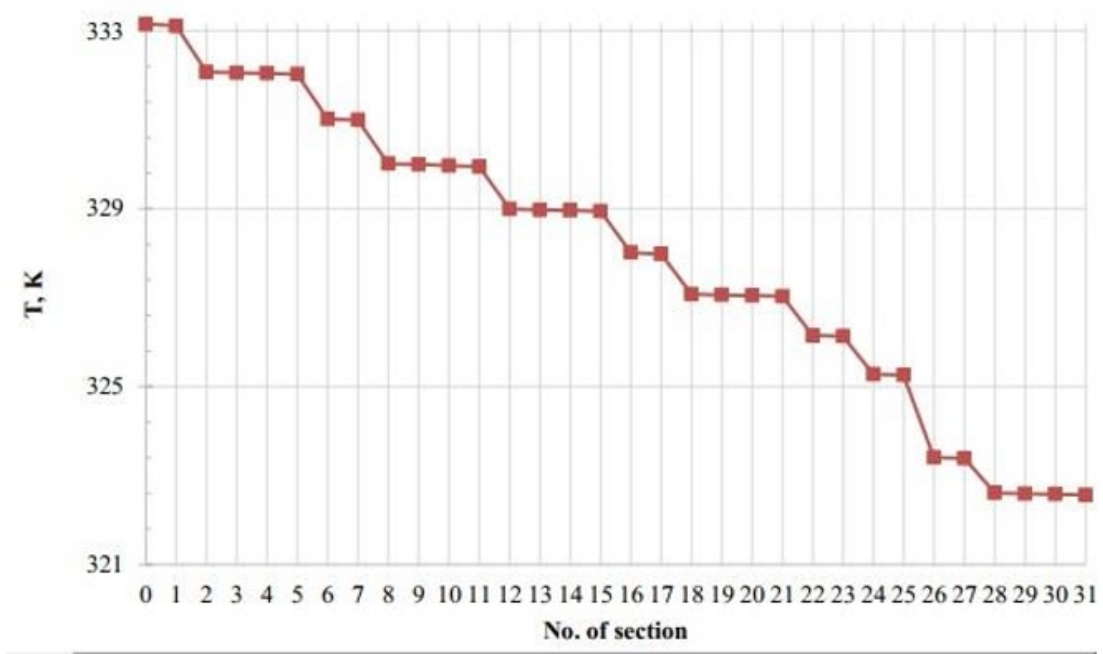

Fig. 5. Fluid temperature change inside isolated parts of the pipeline for the test 1 .

Heat losses q were evaluated according to the equation for the resulting temperature distribution:

$$
q=\frac{\left(T-T_{e n v}\right)}{R_{\Sigma}}
$$

The value $R_{\Sigma}$ here denotes thermal resistance. Cumulative heat loss through the whole pipeline was $8.8 \%$.

Thereafter, the computed curves were compared to the experiment results from [3] (figure 4). It was found that the relative difference between calculated and experimental data for the pressure from [3] was not higher than $4 \%$. 


\subsection{Test 2: System of horizontal pipes}

For the second test, which premise was to restore the initial temperature and pressure values basing on the known data at the pipeline outlet, a branch that was made of 10 consecutive 100 meters long horizontal pipes was taken. The known pressure at the end of the pipeline was $p_{10}=0.1 \mathrm{MPa}$, the temperature $T_{10}=322 \mathrm{~K}$, the mass flow $\mathrm{G}=136.63 \mathrm{~kg} /(\mathrm{m} 2 \mathrm{~s})$, the diameter of straight pipes $\mathrm{d}=0.301 \mathrm{~m}$.

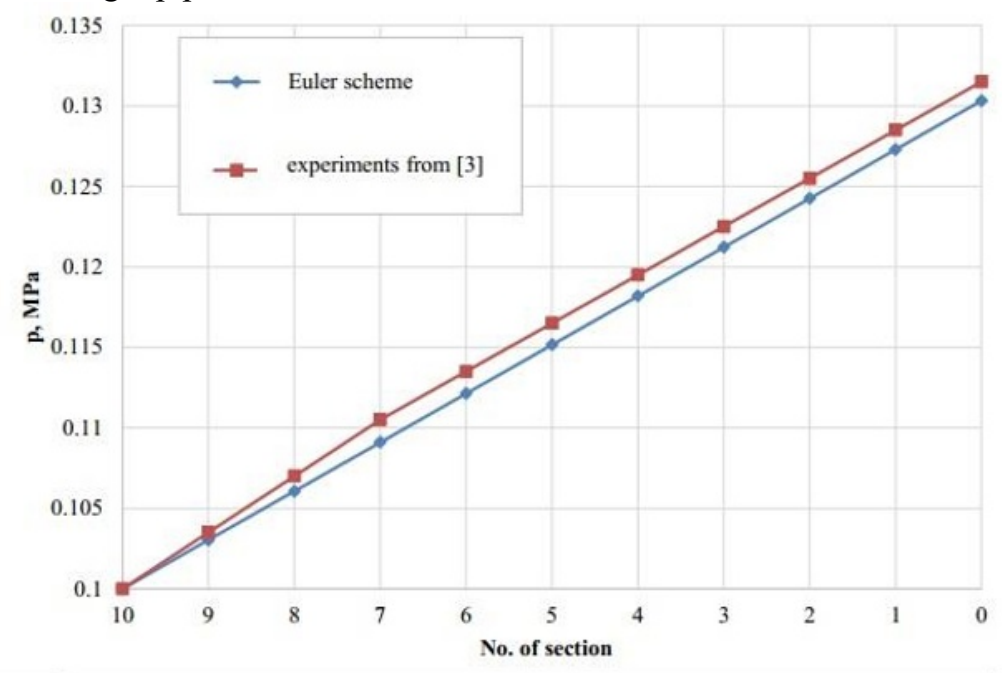

Fig. 6. Restoratio of the initial pressure in the test 2 .

Restoration of the initial values was done by solving the system (1)-(2) using the Euler method with 'reversed time'. In that case p10 and T10, were set as the initial values which means the starting point was moved to the end of the branch and a length increment $\Delta l$ was set to be negative.

Reverse solution for the pressure in the current test is presented in the figure 6. Restored value corresponds to the point at 0 . The same graph has the experimental data of the pressure change. As seen from the figure, relative difference between the value of $\mathrm{p} 0$ calculated using Euler method and the value of $\mathrm{p} 0$ from [3] is $0.76 \%$.

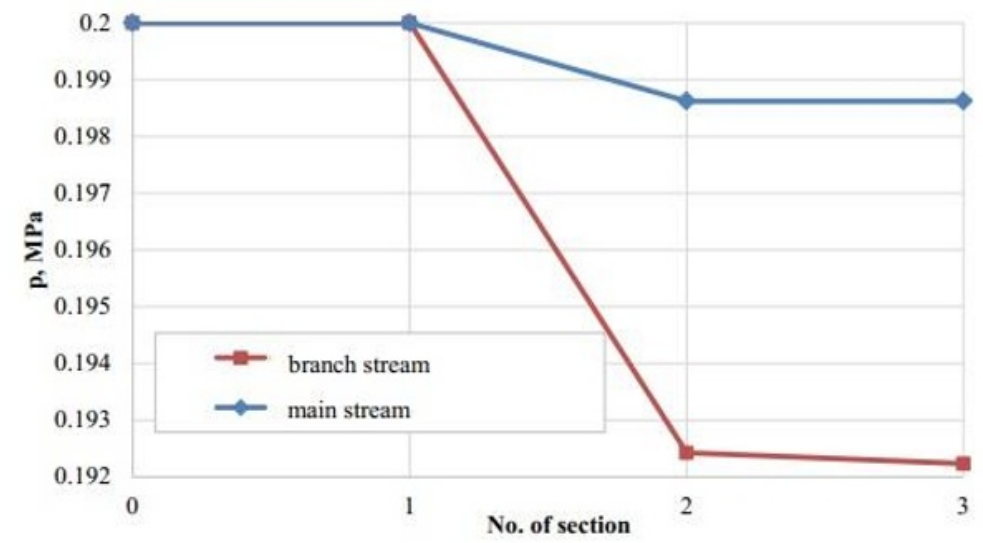

Fig. 7. Pressure change in the pipeline with branching for the test 3 . 


\subsection{Test 3: Branching pipeline}

Tested model for the third example was a pipeline system that contains branchings (figure 2c). All straight pipes had the length of 10 meters. Initial pressure was set to $p_{0}=0.2 \mathrm{MPa}$, initial temperature $T_{0}=333 \mathrm{~K}$, mass flow $\mathrm{G}=117.37 \mathrm{~kg} /(\mathrm{m} 2 \mathrm{~s})$, diameter of straight pipes $\mathrm{d}=0.301 \mathrm{~m}$.

Graphs of pressure drop along primary (section 1-2 in the figure 2c) and offshoot (section 1-3 in the figure 2c) passages are shown in the figure 7 . According to the figure 7 , the pressure losses in the offshoot passage are much larger than in the primary. The cause for this is the higher local resistance in a branched section compared to straight, as well as the influence of hydrostatics.

\section{Conclusion}

Here are the main conclusions on the testing of the program as a short summary.

The model (1)-(2) was implemented in the program using Euler and Runge-Kutta methods, the computational error for each of those methods was found. Result show that at the length increment of $1 \mathrm{~m}$ the relative error was around 10-6 for the Euler and 10-12 for the Runge-Kutta method. Three test models of industrial pipeline systems were designed and used to calculate thermohydraulic properties: graphs of pressure and temperature change through differently oriented parts of the pipelines were plotted; the pressure losses due to the friction inside straight pipes, and local resistances when the pipe becomes narrower/wider and in the places of branching were evaluated; heat losses were calculated. Using the Euler method that allows reversing of time the problem of initial state restoration, knowing values on the pipeline outlet, was solved.

To assess the adequacy of computed results they were compared to the experimental data from external sources. All the comparisons have shown an acceptable difference that does not exceed $1-4 \%$.

\section{Acknowledgements}

The reported study was funded by "Information Technologies Company, Incorporated" according to the contract agreement No A2311030220 (Feb 03, 2020).

\section{References}

1. Yudovina E F, Korelstein L B 2017 Pipeline fittings and equipment 676

2. Novitsky N N, Sukharev M G, Sardanashvily S A and etc. 2014 Pipeline systems of energetics: Mathematical and computer modeling (Novosibirsk: Melentiev ESI Branch of RAS) p 274

3. Babenko A V, Korelstein L B, Gartman T N 2012 Khimicheskaya Tekhnologiya 7429

4. Babenko A V, Korelstein L B, Gartman T N 2012 Oil and Gas Technologies 333

5. Babenko A V, Yudovina E F, Korelstein L B 2019 Neft. Gas. Novacii 964

6. Babenko A V, Yudovina E F, Korelstein L B, Gartman T N 2013 Software \& Systems 1141

7. Likhachev E R 2014 Proceedings of Voronezh State University. Series: Physics. Mathematics 341

8. Kiselev P G 1972 Hydraulic calculations handbook (Moskow: Energy) p 312 
9. Ageeva V V 2011 Hydraulic. Part 2. Hydrodynamics (Nizhny Novgorod: NNSAGU) p 81 